\title{
ADAPTACIÓN DE INDICADORES DE TURISMO SOSTENIBLE: IMPLEMENTACIÓN EN CHIAPAS, MÉXICO
}

\section{ADAPTATION OF SUSTAINABLE TOURISM INDICATORS: IMPLEMENTATION IN CHIAPAS, MEXICO}

\author{
Tamara Rioja-Paradela ${ }^{1}$ \\ Edali Camacho-Ruiz ${ }^{2}$ \\ Arturo Carrillo-Reyes ${ }^{3}$ \\ Eduardo Espinoza-Medinilla ${ }^{4}$ \\ Laura Porras-Murillo ${ }^{5}$
}

\begin{tabular}{|l|l}
\hline Recibido: 26.02 .20 & Aprobado: 29.06 .20
\end{tabular}

DOI: $10.15517 /$ isucr.v21i44.43943

\section{Resumen}

Este estudio adapta y propone una serie de indicadores de turismo sostenible, así como un índice de sostenibilidad turística. Se aplicaron indicadores cuantitativos y cualitativos en un centro turístico de Chiapas, México. De manera relevante se identifican los aspectos críticos que deben abordarse para incrementar la sostenibilidad, y se detalla indicadores que pueden ser utilizados por los administradores del sitio y otros proyectos turísticos similares.

Palabras clave: ecoturismo; indicadores; medición; México; turismo sostenible.

\section{Abstract}

This study adapts and proposes a series of sustainable tourism indicators, as well as a tourism sustainability index. Quantitative and qualitative indicators were applied in a tourist center of Chiapas, Mexico. In a relevant way, this study identifies the critical aspects that must be addressed to increase sustainability and details indicators that can be used by the site administrators and in similar touristic proyects.

Keywords: ecotourism; indicators; measurement; Mexico; sustainable tourism.

\footnotetext{
${ }^{1}$ Mexicana. Profesora-Investigadora, Cuerpo Académico Sustentabilidad y Ecología Aplicada, Universidad de Ciencias y Artes de Chiapas, México. Correo electrónico: tamara.rioja@unicach.mx

${ }^{2}$ Mexicana. Profesora-Investigadora, Universidad de Ciencias y Artes de Chiapas, México. Correo electrónico: edali.camacho@unicach.mx

${ }^{3}$ Mexicano. Profesor-Investigador, Cuerpo Académico Sustentabilidad y Ecología Aplicada, Universidad de Ciencias y Artes de Chiapas, México. Correo electrónico: arturo.carrillo@unicach.mx

${ }^{4}$ Mexicano. Profesor-Investigador, Cuerpo Académico Sustentabilidad y Ecología Aplicada, Universidad de Ciencias y Artes de Chiapas, México. Correo electrónico: eduardo.espinoza@unicach.mx

${ }^{5}$ Costarricense. Instituto Internacional de Conservación y Manejo de Vida Silvestre, Universidad Nacional de Costa

Rica. Correo electrónico: laura.porras.murillo@una.cr
} 


\section{Introducción}

El turismo es una de las principales actividades que contribuyen al crecimiento y la diversificación económica de un país o localidad (World Tourism Organization, 2004). Este puede generar impactos positivos en los niveles ambiental, social, económico y cultural; no obstante, también pueden ser impactos negativos si no existe una adecuada planeación (Habibullah, Din, Chong, \& Radam, 2016). En ese contexto que se vuelve indispensable que el turismo se desarrolle bajo preceptos de sostenibilidad, de modo que la generación de ingresos provoque un impacto mínimo en el ambiente y en la identidad cultural de las comunidades locales (Pérez Albert \& NelIo Endreu, 2013).

Actualmente, la protección del ambiente natural y social como parte de un modelo de turismo sostenible es un punto fundamental para el incremento de la competitividad turística (Blancas-Peral, González-Lozano, Guerrero-Casas, \& Lozano-Oyola, 2010), particularmente en sitios cercanos a áreas naturales protegidas (Blanco-Cerradelo, Diéguez-Castrillón, \& GueimondeCanto, 2015). La necesidad de mantener el capital natural y sociocultural de los destinos turísticos permite ver más allá de una actividad basada netamente en el ámbito económico (Herrero, 2017; Luffiego-García \& Rabadán-Vergara, 2000). Por ello, han surgido diversas propuestas para desarrollar instrumentos que permitan la medición precisa de los avances en este modelo de sostenibilidad del sector (Pérez Albert \& Nel-Io Endreu, 2013). En ese sentido, a fin de medir las afectaciones de la actividad turística y su acercamiento al paradigma de la sostenibilidad se ha impulsado a nivel internacional el uso de indicadores (World Tourism Organization, 1996, 2004), que buscan generar puntos de referencia que ubiquen el estado de sostenibilidad de proyectos turísticos (Camacho-Ruiz, Carrillo-Reyes, Rioja-Paradela, \& Espinoza-Medinilla, 2016). En ese contexto, se propuso medir la sostenibilidad en el manejo y operación del Centro Turístico Sima de las Cotorras [CTSC], en Chiapas, México, mediante la aplicación de un sistema de indicadores diseñados para tal efecto. Se pretende que los resultados de la evaluación permitan encausar estrategias así como reorientar las actividades del destino, para conservar y proteger los recursos naturales, y asegurar una experiencia satisfactoria para los visitantes. 


\section{Metodología}

Área de estudio.- El Complejo Turístico Sima de las Cotorras (STSC) se localiza en el área de influencia del área natural protegida Reserva de la Biosfera Selva El Ocote [REBISO] en el municipio de Ocozocoautla de Espinosa, Chiapas, México (Figura 1).

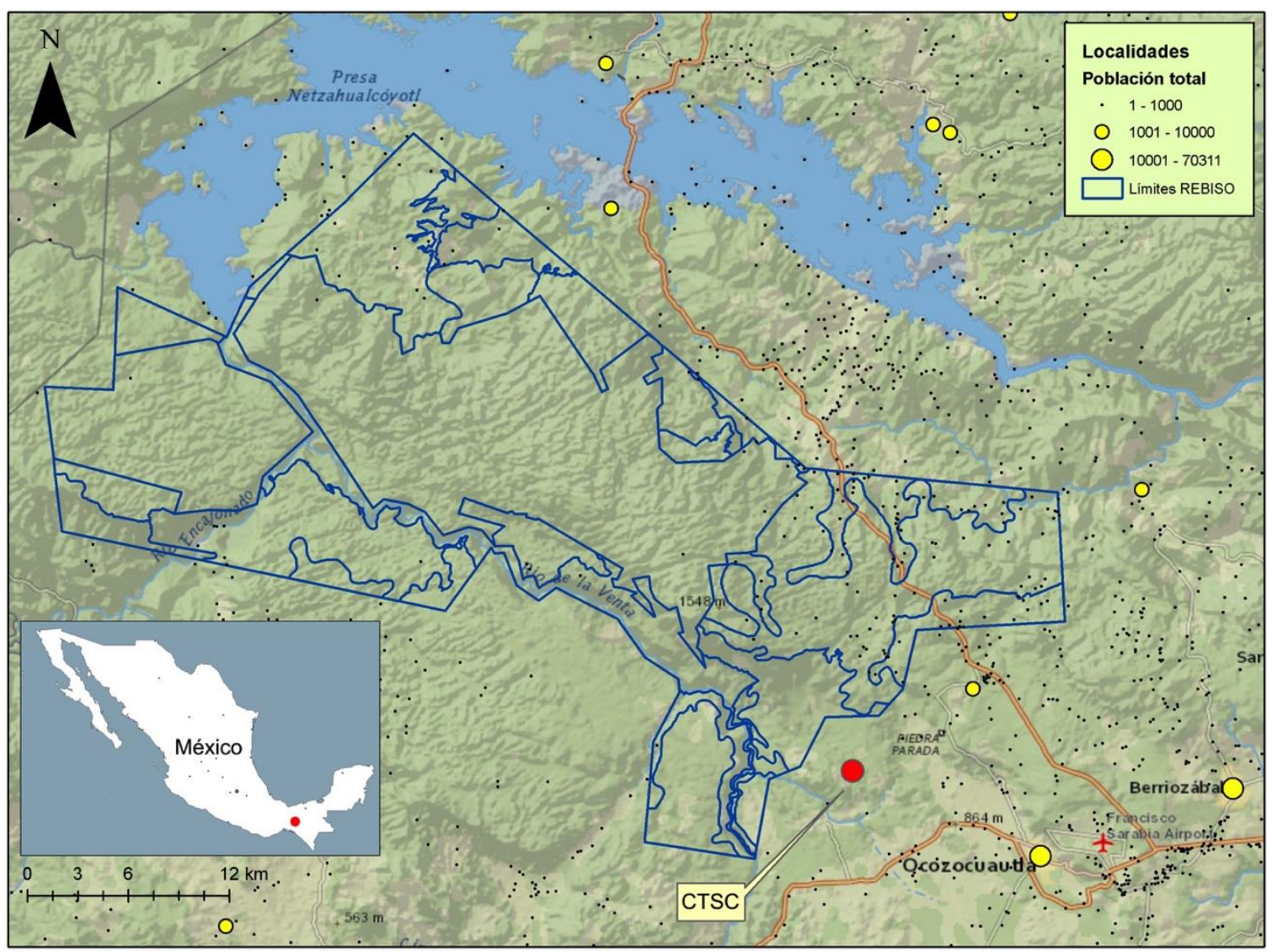

Figura 1. Ubicación del Centro Turístico Sima de las Cotorras (punto rojo), al sur de la Reserva de la Biósfera Selva El Ocote. Imagen de fondo: World Terrain Base (ESRI, 2009).

Es una zona de clima cálido con lluvias en verano, y está dominada por selvas secas y vegetación secundaria (Alarcón Hernández, 2010; Secretaría de Medio Ambiente y Recursos Naturales, 2001). El CTSC tiene como principal atractivo una dolina, formación geológica de 150 metros de diámetro y 145 de profundidad, en cuyas paredes descansan durante la noche cientos de cotorras verdes (Aratinga holochlora), una especie en peligro de extinción (Alarcón Hernández, 2010; Secretaría de Medio Ambiente y Recursos Naturales, 2001).

El CTSC es administrado por la Cooperativa Tzamanguimó, misma que pertenece a la Red de Ecoturismo Pluriétnico de la Selva El Ocote. Los miembros de esta cooperativa son originarios de la localidad rural Rivera Piedra Parada, la que cuenta con 435 habitantes. La comunidad se 
clasifica en niveles de mediana a alta marginación (Instituto Nacional de Estadística y Geografía, 2010). El estudio se realizó con el permiso de los administradores del sitio y la comunidad (CanoContreras, Medinaceli, Diago, \& Villamar, 2015).

El estudio se realizó en dos temporadas altas (julio y diciembre) y dos bajas (septiembreoctubre y febrero), definidas en función de la intensidad de la actividad turística de acuerdo a los registros del centro, quienes reportan hasta 3,500 visitantes en promedio por temporada alta y 400 visitantes en promedio por temporada baja. El estudio se realizó en ambas temporadas debido a que la estacionalidad del flujo de visitantes modifica el resultado de los indicadores de sostenibilidad turística.

Sistema de indicadores.- La falta de un sistema de indicadores estandarizado permite a cada destino turístico desarrollar y adaptar indicadores propios. Este estudio se basó en la propuesta de Rivas y Magadán (2007), quienes utilizaron herramientas creadas por la Organización Mundial de Turismo [OMT] (World Tourism Organization, 1996, 2004) e indicadores propuestos por Vera-Rebollo \& Baidal (2003).

Se identificaron los atributos y los temas prioritarios del sitio para los aspectos económicos, sociales y ambientales. Los atributos son el conjunto de características que proporcionan la base contra la cual se midieron los indicadores a través del tiempo (World Tourism Organization, 1996, 2004). Una vez identificados y siguiendo la propuesta de la OMT se procedió a su selección, asignando una ponderación a cada atributo identificado en una escala ascendente de 1 a 10, en función del nivel de sensibilidad del atributo al impacto del turismo, siendo 10 la máxima sensibilidad. Posteriormente, se identificaron los temas prioritarios o clave. Para ello, y mediante talleres con los manejadores del sitio, se revisaron los indicadores propuestos por Rivas \& Magadán (2007), buscando responder a las siguientes preguntas: ¿Los indicadores incluyen la mayoría o una parte importante de los temas prioritarios? $\mathrm{Si}$ no es así, ¿Qué medidas o indicadores complementarios del sitio son necesarios para proporcionar la información requerida para esos temas?. La propuesta de indicadores complementarios propios del área se realizó siguiendo el marco de evaluación y criterios propuesto por la OMT de acuerdo a la metodología comunitaria para el desarrollo social MECOM (Kniffki, Calero, \& Castillo, 2009).

Entrevistas semi-estructuradas.- Se aplicaron 462 entrevistas semi-estructuradas a visitantes y habitantes de la zona que accedieron a colaborar. Durante las temporadas bajas se entrevistaron 
124 personas, y durante las temporadas altas se entrevistaron 338 personas. Con estas se obtuvo información sobre la percepción del sitio de visitantes y pobladores de la zona, necesario para responder a los indicadores.

Observación participante.- Mediante ésta se obtuvo información sobre la opinión de la comunidad con respecto a la actividad ecoturística, incremento en la conflictividad, accidentes de tráfico y nivel de instrucción. Dichos datos se obtuvieron a partir de charlas y reuniones sostenidas con los gestores del centro y habitantes de la comunidad.

Selección y adaptación de indicadores.- La selección y evaluación de indicadores básicos y complementarios se basó en los criterios generales de evaluación propuestos por la OMT (World Tourism Organization, 1996, 2004), que incluye: a) obtención de datos e información: estimado de la facilidad para obtener la información, b) indicadores verosímiles y fácilmente interpretables: se seleccionaron indicadores fiables para todo tipo de público, c) indicadores que permitan predecir tendencias en el tiempo y la comparación entre áreas, d) indicadores predictivos de sostenibilidad: grado en que proporcionan información sobre la sostenibilidad ambiental, económica y social, y finalmente e) disponibilidad de valores de entrada y referencia, es decir, indicadores con información de referencia para evaluar la tendencia general de los mismos.

Aplicación de los indicadores.- Se propone la siguiente adaptación a la propuesta de Rivas \& Magadán (2007), incluyendo un índice de aplicación simple que integra la totalidad de los indicadores y establece un valor de referencia, denominado aquí como índice de sostenibilidad turística [IST]. Se asume una valoración similar para todos los indicadores, es decir, todos los indicadores contribuyen con el índice final en la misma proporción.

Dado que Rivas \& Magadán (2007) no propusieron un parámetro de comparación, este trabajo se realizó considerando los valores óptimos de cada indicador como el parámetro de referencia. Para el caso de este estudio, el valor máximo del IST es de 1250, cuando todos los indicadores alcanzan su valor óptimo. El cálculo de cada indicador, se realizó de acuerdo a lo propuesto por los autores, con excepción de dos indicadores para los que se hace un ajuste descrito más adelante:

donde:

IST = Índice de sostenibilidad turística 
Indicadores ambientales

$\mathrm{a}=$ Cobertura vegetal

$\mathrm{b}=$ Percepción de la calidad visual

$\mathrm{c}=$ Contaminación visual

$\mathrm{d}=$ Deterioro del paisaje natural y rural

e $=$ Expansión del área turística construida

$\mathrm{f}=$ Presencia de especies endémicas

$\mathrm{g}=$ Disponibilidad de agua limpia para proyectos turísticos

$\mathrm{h}=$ Contaminación auditiva percibida por los turistas

$\mathrm{i}=$ Congestión vehicular

$\mathrm{j}=$ Calidad ambiental de proyectos

$\mathrm{k}=$ Deterioro de estructuras de interés

Indicadores económicos:

1 = Ingreso generado por empleo generado por actividades turísticas

$\mathrm{m}=$ Grado de satisfacción en servicios turísticos

$\mathrm{n}=$ Volumen de turistas en periodo de alta demanda

o = Nivel de ocupación de instalaciones turísticas

$\mathrm{p}=$ Diversificación de productos turísticos

$\mathrm{q}=$ Empleo directo total generado por el turismo

$r=$ Variación estacional del empleo

$\mathrm{s}=$ Licencias municipales

Indicadores socioculturales:

$\mathrm{t}=$ Relación visitantes/residentes

$\mathrm{u}=$ Percepción de la comunidad acerca del turismo

$\mathrm{v}=$ Incremento del conflicto o crimen en temporada alta

$\mathrm{w}=$ Accidentes de tráfico en temporada alta

$\mathrm{x}=$ Nivel educativo de trabajadores del sector turístico 
Para los indicadores " $\mathrm{n}$ " $\mathrm{y}$ " $\mathrm{t}$ " se tomaron las siguientes consideraciones, con el fin de que se ajustaran al índice general:

cuando $n<=50$, entonces $n=n$

si $n>50$, entonces $(n-100)(-1)$

si $\mathrm{t}<=100$, entonces $t=t$

si $t>100$, entonces $((1-t) * 100)(-1)$

\section{Resultados y discusión}

El sistema de indicadores de sostenibilidad turística para el CTSC se compuso de 15 indicadores ambientales, ocho económicos y seis socioculturales. Los criterios decisivos fueron la obtención de información y los valores de entrada, seguidos del criterio de temporalidad y credibilidad. Finalmente, se obtuvieron seis indicadores complementarios. Al aplicar la ecuación propuesta, se encontró que el CTSC se encuentra lejos del valor óptimo de sostenibilidad, con un $I S T=362.65$. Al analizar el CTSC por temporada, el resultado fue similar, con un IST para la temporada alta de 448.24 y de 436.08 para la temporada baja (Cuadro 1).

Cuadro 1. Resultado de los indicadores evaluados por temporada y anualmente, así como el valor óptimo por cada indicador

\begin{tabular}{|c|c|c|c|c|c|}
\hline Dimensión & Indicador & Alta & Baja & Anual & Óptimo \\
\hline \multirow[t]{11}{*}{ Ambiental } & Cobertura vegetal con deterioro. & \multicolumn{2}{|c|}{58.78} & 58.78 & 0 \\
\hline & Percepción de la calidad visual. & 5.40 & 1.33 & 3.33 & 0 \\
\hline & Contaminación visual. & 0 & 0 & 0 & 0 \\
\hline & Deterioro del paisaje natural y rural. & \multicolumn{2}{|c|}{0.90} & 0.90 & 0 \\
\hline & $\begin{array}{l}\text { Expansión del espacio turístico } \\
\text { construido. }\end{array}$ & 0 & 0 & 0 & 0 \\
\hline & Presencia de especies endémicas & $*$ & $*$ & * & 100 \\
\hline & $\begin{array}{l}\text { Disponibilidad de agua dulce para } \\
\text { nuevos proyectos turísticos. }\end{array}$ & \multicolumn{2}{|c|}{0} & 0 & 100 \\
\hline & $\begin{array}{l}\text { Contaminación acústica de fuentes } \\
\text { móviles percibida por turistas. }\end{array}$ & 0 & 0 & 0 & 0 \\
\hline & Congestión vehicular. & \multicolumn{2}{|c|}{1.12} & 1.12 & 0 \\
\hline & Calidad ambiental de los proyectos. & $*$ & $*$ & $*$ & 100 \\
\hline & $\begin{array}{l}\text { Deterioro de estructuras de interés } \\
\text { turístico. }\end{array}$ & $*$ & $*$ & $*$ & 0 \\
\hline \multirow[t]{2}{*}{ ECONÓMICOS } & $\begin{array}{llll}\text { Ingresos } & \text { generados por empleo } \\
\text { turístico. } & & & \\
\end{array}$ & \multicolumn{2}{|c|}{50.84} & 61.36 & 100 \\
\hline & $\begin{array}{l}\text { Grado de satisfacción en servicios } \\
\text { turísticos. }\end{array}$ & 26.62 & 11.78 & 19.20 & 100 \\
\hline
\end{tabular}




\begin{tabular}{|c|c|c|c|c|c|}
\hline & $\begin{array}{l}\text { Volumen de turistas en período de } \\
\text { demanda alta. }\end{array}$ & \multicolumn{2}{|c|}{77.05} & 77.05 & 50 \\
\hline & $\begin{array}{l}\text { Nivel de ocupación de alojamientos } \\
\text { turísticos. }\end{array}$ & $*$ & $*$ & $*$ & 100 \\
\hline & $\begin{array}{l}\text { Diversificación del producto } \\
\text { turístico. }\end{array}$ & \multicolumn{2}{|c|}{66.66} & 66.66 & 100 \\
\hline & $\begin{array}{l}\text { Total de empleo directo generado } \\
\text { por el turismo. }\end{array}$ & 5.86 & 4.46 & 5.86 & 100 \\
\hline & Variación del empleo. & \multicolumn{2}{|c|}{76.19} & 76.19 & 100 \\
\hline & Licencias municipales. & $*$ & $*$ & * & 100 \\
\hline \multirow[t]{5}{*}{ SOCIOCULTURALES } & $\begin{array}{l}\text { Relación } \\
\text { residente. }\end{array}$ & \multicolumn{2}{|c|}{77.24} & 77.24 & 100 \\
\hline & $\begin{array}{l}\text { Percepción de la comunidad a cerca } \\
\text { de la actividad turística. }\end{array}$ & \multicolumn{2}{|c|}{96.15} & 96.15 & 100 \\
\hline & $\begin{array}{l}\text { Incremento de la conflictividad y el } \\
\text { delito en temporada alta. }\end{array}$ & 0 & 0 & 0 & 0 \\
\hline & $\begin{array}{l}\text { Accidentes de tráfico en temporada } \\
\text { alta. }\end{array}$ & 0 & 0 & 0 & 0 \\
\hline & $\begin{array}{l}\text { Nivel de instrucción de los } \\
\text { trabajadores de la actividad turística. }\end{array}$ & \multicolumn{2}{|c|}{29.5} & 29.5 & 100 \\
\hline
\end{tabular}

* Sin datos

Este resultado está determinado por los indicadores con valores bajos con respecto al óptimo, tales como el volumen de turistas en temporada alta, el empleo total generado por la actividad turística, el nivel educativo de los empleados de la actividad turística y el deterioro de la cobertura vegetal.

El resultado del volumen de turistas en temporada alta indica una alta estacionalidad $(77 \%)$ con respecto a su valor óptimo, que es aquel en el que la llegada de turistas no es marcadamente estacional. En cuanto al empleo generado por la actividad turística, se encontró que el CTSC genera el $5.86 \%$ de empleos con respecto al $100 \%$ de empleos para la comunidad. El índice de nivel instrucción obtuvo un resultado del 29.5\%, indicando que únicamente ese porcentaje de trabajadores con respecto al total cuenta con el nivel educativo básico. Por último, el valor del $58.78 \%$ en el índice de cobertura vegetal muestra que la superficie correspondiente a ese valor porcentual sobrepasa el óptimo, lo que señala deterioro existente en el área del CTSC. Por otro lado existen indicadores como, presencia de especies endémicas, deterioro de estructuras de interés turístico y nivel de ocupación de alojamientos para los cuales no existieron valores medibles por la ausencia de información disponible para el área de estudio. De manera que estos indicadores sin valores porcentuales adicionales a los indicadores detallados en el párrafo anterior incidieron sobre un valor bajo en el IST global. 


\section{Indicadores básicos}

Cobertura vegetal.- Dentro de la valoración cualitativa total del territorio, se encuentra la valoración ecológica. En ella es posible aplicar criterios de evaluación a través de Mapas de Uso del Suelo y Cobertura Vegetal para clasificar un sitio desde muy alto hasta sin valor ecológico (Chuvieco, 1998). Dado a la carente información de salida del sitio para la elaboración de mapas de valor ecológico, se propone utilizar la medición de la cobertura vegetal. El resultado de la medición del indicador de cobertura vegetal revela que 5.21 Ha de 8.8 Ha disponibles, muestran una marcada evidencia de alteración en el CTSC. Es decir, no hay evidencia de que se de uso óptimo a los recursos naturales para mantener los procesos ecológicos esenciales, conservar, planificar y manejar adecuadamente la diversidad biológica (Vanegas-Montes, 2006).

Percepción de la calidad visual.- Un espacio territorial posee características básicas del paisaje que posibilitan reconocer su valoración paisajística de acuerdo a parámetros como la unidad visual, organización visual, calidad visual y calidad escénica. La calidad visual del paisaje representa la condición primaria a partir de la cual es posible construir otros valores más complejos como la fragilidad, capacidad de absorción visual e impacto visual (Solari \& Cazorla, 2009). La medición de este indicador fue cualitativa, a través de entrevistas a los visitantes con respecto a su percepción de la diversidad biofísica, naturalidad, singularidad, complejidad topográfica, actividades humanas y degradación del área en estudio. El valor de este indicador fue del 3.33\%. Es decir, de acuerdo a los visitantes, la percepción de la calidad visual del CTSC es cercana a la óptima. El mantenimiento de este indicador en el mismo nivel puede asegurar el interés de los visitantes, elevando su competitividad y nivel de indicadores ambientales.

Contaminación visual.- Se considera contaminación visual al desequilibrio del paisaje que afecta las condiciones de vida y las funciones vitales de los seres vivientes. Esto se refiere a los efectos perturbadores que afectan en tres niveles: estético-paisajístico, degradación de la calidad del entorno y psicoactivo, produciendo sobrecarga informática o fatiga (Méndez-Velandia, 2013). El índice de contaminación visual en el CTSC muestra un valor anual óptimo del cero por ciento, indicando que el total de visitantes anuales entrevistados señalaron no percibir contaminación visual producida por exceso de señalamientos y/o residuos sólidos dentro del centro.

Deterioro del paisaje natural y rural.- En las etapas de desarrollo y operación de los proyectos turísticos se presentan actividades que provocan alteraciones sobre los recursos naturales y culturales, entre ellos la superficie construida (Rivas, 1998). Para el caso de estudio, este indicador 
muestra un porcentaje menor del $1 \%$ con respecto al óptimo de $0 \%$. El resultado de este indicador es positivo y parece evidenciar que el impacto al ambiente por superficie construida ha sido mínimo. Es deseable mantener su valor cercano al óptimo para mantener las áreas naturales que permitan mantener en el tiempo zonas factibles de permanencia de la biodiversidad (Rivas, 1998). Expansión del espacio turístico construido.- Indica la superficie en metros cuadrados en que la infraestructura se ha incrementado en el área de estudio con respecto a cierto periodo de tiempo. Se estableció un periodo de dos años como el óptimo debido a que es el tiempo propicio para generar proyectos de infraestructura. El resultado de este indicador es el óptimo dado que la satisfacción de los turistas con respecto a la infraestructura en el centro fue alta. Desde una perspectiva ambiental estricta permite asegurar el cumplimiento de los ejes rectores del ecoturismo sostenible (Vanegas-Montes, 2006), por lo que se demuestra cuantitativamente que las condiciones de infraestructura actuales en el centro son suficientes y no es necesario incrementarla. Sin embargo, debe señalarse que en este periodo de tiempo no se ha llevado a cabo proyecto alguno que considere el establecimiento de nuevos espacios que permitan la diversificación de actividades recreativas y que a su vez cumplan con los criterios del ecoturismo. La planificación y ejecución de estas demandas requiere de un análisis que permita conocer cuantitativamente la superficie máxima permisible en el área y la capacidad de carga del ecosistema (Pavón, Muñoz, \& Arcos, 2007), entendida esta última como la facultad del ambiente a resistir y adaptarse a los cambios externos (Vera-Rebollo \& Baidal, 2003).

Presencia de especies endémicas.- Para la zona de estudio no existen datos de este indicador. Adicionalmente, se propone la simplificación de la medición de este indicador, eliminando la medición de la frecuencia relativa propuesta por Rivas \& Magadán (2007), lo que facilitaría su medición. Se propone el siguiente ajuste:

donde:

IPE: Índice de persistencia de especies endémicas

$\mathrm{e}_{2}$ : Número de especies endémicas actuales

$\mathrm{e}_{1}$ : Número de especies endémicas en un periodo anterior

Esta propuesta cuantifica la persistencia de especies endémicas sin la inclusión de factores de extinción de acuerdo a lo citado por los mismos autores. También se propone como medición 
óptima el valor de $100 \%$, valor que indicaría la permanencia del total de especies endémicas a través de un periodo de tiempo. Este indicador puede mantener valores altos cuando se mantienen otros indicadores tales como el de cobertura vegetal o el de contaminación auditiva, aspectos con una relación directa con la presencia de fauna silvestre.

Disponibilidad de agua dulce para nuevos proyectos turísticos.- El tema del agua en el sector turístico es estratégico, considerando los retos de disponibilidad, gestión integral y tratamiento del mismo (Gössling et al., 2012; World Tourism Organization, 2004). Por ello, la disponibilidad del agua compromete la actividad turística y su medición debe ser prioritaria. Para el área de estudio, no existe acceso directo al agua, por lo que el abastecimiento del recurso es a través de su transporte desde un sitio diferente al CTSC. Por ello el resultado de su medición es cero. Una propuesta es elevar el valor de este indicador mediante la implementación de proyectos basados en ecotecnologías que permitan la captación y almacenamiento de agua lluvia. Los sistemas de captación de agua pluvial (CAP) permiten abastecer de agua para la vida rural y urbana (Neibaur \& Anderson, 2016). Además permiten disminuir la presión sobre los acuíferos y proyectos de construcción de nuevas presas para el abastecimiento (Gleason, 2005). En este sentido, se propone considerar el almacenamiento como "recurso disponible inicial" para efectos de la medición del indicador. También se hace necesario incluir un factor de medición sobre la tasa de consumo del recurso tanto en temporada alta como en la baja, o bien, que se construya un indicador complementario específico para tal efecto.

Contaminación acústica por fuentes móviles percibida por los turistas.- La contaminación acústica es otro de los retos en los destinos turísticos; esta perturba las actividades comunitarias, interfiere en la comunicación hablada, puede causar perturbaciones del sueño y el descanso, impide la concentración y el aprendizaje, y crea estados de cansancio y tensión en los visitantes de los destinos turísticos (Mihalič, 2000; Stansfeld \& Matheson, 2003). También puede ser causa de perturbación a las poblaciones animales (Barber, Crooks, \& Fristrup, 2010; Iglesias, Mata, \& Malo, 2012) lo que podría derivar no solo en reducir la presencia de fauna silvestre, sino incluso ahuyentar al atractivo más importante del sitio, la cotorra verde. Este indicador muestra un valor óptimo para el CTSC. Se propone medir el indicador de contaminación acústica utilizando un decibelímetro, lo que arrojaría datos concretos y fácilmente comparables.

Congestión vehicular.- El turismo puede relacionarse con fenómenos como la congestión vehicular. Mendoza-Ontiveros, Monterrubio-Cordero, \& Fernández-Aldecua (2011) señalan que 
el turismo aumenta el número de habitantes, congestión vehicular, ruido y basura. Para el CTSC este indicador presentó un valor de 1.12, indicando que la percepción de visitantes y residentes de la comunidad es positiva, dado que este factor no les genera incomodidad y tampoco perciben alteraciones en su intimidad como comunidad.

Calidad ambiental de los proyectos.- La calidad es un proceso decisivo para la competitividad de los destinos turísticos. Hassan (2000:240) define competitividad turística como "la capacidad de un destino para crear e integrar productos con valor añadido que permitan sostener los recursos locales y conservar su posición de mercado respecto a sus competidores". En la actualidad la incorporación de proyectos de calidad ambiental en los destinos turísticos permite implementar prácticas ambientales que contribuyen al desarrollo de un turismo responsable y sostenible (Ayuso Siart, 2003). Dichas prácticas se dirigen en primer lugar a la dimensión ambiental del desarrollo sostenible pero además incluyen cuestiones sociales y económicas (World Tourism Organization, 2004). Para esta investigación no se contó con información para el indicador, debido a la falta de información disponible sobre proyectos realizados en el sitio. No obstante, es importante iniciar la recopilación de datos, de modo que los gestores del destino cuantifiquen su avance y posicionamiento en la competitividad ambiental y social con destinos similares.

Deterioro de estructuras de interés turístico.- Este indicador se relaciona con la percepción de seguridad y limpieza de los destinos turísticos (Lemus \& Pérez, 2011). Dado que Rivas \& Magadán (2007) no definen su concepto de deterioro, en este estudio se propone que este indicador se calcule como el valor porcentual del número de personas que mencionan percibir deterioro de la estructura en relación al número total de entrevistados.

\section{Indicadores económicos}

Ingresos generados por empleo turístico.- Una atributo fundamental que relaciona la actividad turística y a los administradores de los mismos son los ingresos generados (Rivas García \& Magadán Díaz, 2007). En el sitio de estudio, las actividades turísticas no representan el 100\% de los ingresos de quienes lo administran. El indicador muestra que al menos el 50\% de los ingresos totales de cada uno de los gestores del CTSC corresponde a la actividad turística en relación al total de actividades económicas que realizan. Un incremento sostenido en este valor implicaría un aumento en la viabilidad económica del sitio. Adicionalmente, lo convertiría en un sector 
económicamente atractivo no solo para quienes ya laboran en el mismo, sino para otros habitantes de la comunidad y sus alrededores.

Grado de satisfacción en servicios turísticos.- Uno de los objetivos del turismo es garantizar la satisfacción de visitantes, pues al no hacerlo se puede generar la caída de los flujos turísticos (Punzo, 2004). Este indicador resultó negativo en el sitio ya que únicamente el 19\% del total de visitantes encuestados repitieron su visita al centro en el periodo de un año. Además, este porcentaje que visita al menos dos veces en el año el destino manifiesta no quedar satisfecho con los servicios turísticos ofertados. Se hizo énfasis en que las actividades ofertadas están dirigidas a un público joven con habilidades físicas. La ampliación de la oferta turística in situ podría derivarse en una mayor afluencia de visitantes, incluyendo aquellos que repitan sus visitas al mismo. A su vez, esto tendría que concretarse mediante la mejora de la infraestructura disponible y la búsqueda de fuentes de ingreso adicionales. No obstante, es en estos rubros donde debe ponerse especial cuidado, pues el incremento de la infraestructura y con ello del número de visitantes, debe mantenerse a un punto tal que no afecte los indicadores ambientales (Vanegas-Montes, 2006).

Volumen de turistas en periodo de demanda alta.- Una de las características comunes en el turismo es la estacionalidad, en la que ocurre un aprovechamiento intensivo de las zonas turísticas durante pocos meses del año (Rivas, 1998). Este indicador muestra que en el CTSC los beneficios económicos por el flujo de visitantes ocurren mayoritariamente durante la temporada alta, con una marcada estacionalidad. Es posible que esto ocurra debido a la falta de difusión del destino así como a la carencia de un turismo de inclusión social (Conferencia de las Naciones Unidas sobre Comercio y Desarrollo, 2013), ya que los visitantes consideran que el producto turístico ofertado es considerado insuficiente para satisfacer a un público de edades avanzadas. La diversificación del producto así como la habilitación del sitio para recibir visitantes de todas las clases de edad podría modificar este valor así como el indicador correspondiente.

Nivel de ocupación de alojamientos turísticos.- La estacionalidad en los destinos turísticos tiene como inconveniente producir épocas de temporadas bajas en donde el nivel de ocupación disminuye sensiblemente y se produce la denominada "Capacidad Ociosa" (Galle, 2004). Es posible que una mejor difusión del sitio aumente el valor de este indicador. De nueva cuenta, debe considerarse que un incremento en la ocupación no afecte negativamente el resto de indicadores. Considerando la estacionalidad como característica no redituable para los destinos y la sostenibilidad, se estableció para este indicador un valor óptimo del 50 por ciento, lo que implica 
que la afluencia turística es similar entre las temporadas de mayor y menor afluencia. Para el sitio de estudio la falta de un registro confiable imposibilitó su medición, por lo que el seguimiento o registro de información para su posterior medida es una recomendación prioritaria.

Diversificación del producto turístico.- El producto turístico son aquellos atractivos (tangibles o intangibles) que motivan a los visitantes a realizar un desplazamiento para realizar actividades relacionadas con el ambiente y el tiempo libre, y el grado de madurez alcanzado por los destinos turísticos incide sobre la diversificación, ya que permite introducir elementos complementarios a partir de las potencialidades locales (Benur \& Bramwell, 2015). Por todo ello, este indicador considera las actividades actuales y potenciales del destino. Estas últimas fueron aquellas registradas a partir de las entrevistas realizadas a los visitantes, en donde se menciona el tipo de actividad que les habría gustado realizar en el destino y que actualmente no se encuentra disponible. Se propone cruzar su resultado con el obtenido del "grado de satisfacción en los destinos turísticos" para la mejor toma de decisiones entre los administradores del centro.

Total de empleo directo generado por el turismo.- La actividad turística crea empleos y cadenas de valor internas. Uno de los retos de este sector es articular los diferentes sectores productivos en un mismo marco territorial para generar sinergias entre ellos. De esta manera se pueden integrar y aprovechar oportunidades y estructuras para construir nuevas realidades sociales y productivas (Ávila-Bercial \& Barrado-Timón, 2005). De acuerdo a Bolwell \& Weinz (2008) el turismo también genera empleos debido a su carácter estacional, mismos que pueden ser deficientes y por tanto no calificados. Según la Organización Internacional del Trabajo (OIT) se estima que por cada empleo se crea aproximadamente 1.5 empleos adicionales en actividades económicas relacionadas con el sector. Para el CTSC el indicador muestra que sólo el 5.86\% del empleo es generado por la actividad turística, es decir, no parece ser redituable para los administradores del destino, por lo que adicionalmente deben realizar otras actividades que contribuyan a incrementar su ingreso económico.

Variación del empleo.- La existencia de empleos estacionales propicia la incapacidad de los destinos de construir ocupaciones permanentes con operadores especializados, se incrementan los costos de búsqueda, contratación y capacitación de personal y por tanto disminuyen la calidad de los servicios del sector (Carruitero, 2011). Para el CTSC este indicador muestra una variación del empleo del $76.19 \%$, indicando claramente una variación temporal del empleo. Una vez más el valor de este indicador se relaciona con el "volumen de turistas en periodo de demanda alta", ya que al 
haber estacionalidad en el destino la contratación de personal varía de igual forma. Tal y como en el caso anterior, se propone la introducción de elementos que permitan la diversificación del producto turístico y que este sea incluyente. Dicha diversificación puede incidir sobre indicadores como variación del empleo, nivel de ocupación y grado de satisfacción en los servicios turísticos. Licencias municipales.- El desarrollo turístico local requiere de licencias municipales. Por tanto el municipio como regulador y administrador del territorio gestiona los permisos en varios ámbitos, como la planificación del uso del suelo, servicios básicos y construcción de la obra pública, regulación y control de desarrollo, recaudación de impuestos y, coordinación y promoción. La proporción de licencias municipales otorgadas a la actividad turística con relación a la totalidad de licencias para el comercio otorgadas por el municipio es un indicador de los propuestos por Rivas \& Magadán (2007) que puede indicar la importancia de la actividad turística a nivel local. Para este estudio, la ausencia de información sobre la cantidad de permisos gestionados y otorgados por el municipio en relación a la actividad turística en la zona de injerencia del CTSC imposibilitó su medición. Se propone un valor óptimo de 100\%, asumiendo el supuesto de que un alto valor del indicador implica que la actividad turística es intensa y cumple con las normas locales que la regulan. Cabe mencionar que el valor del indicador variará de acuerdo a la demanda del mercado, en cuyo caso se deberá establecer un valor óptimo nuevamente. Finalmente en zonas donde la actividad turística es intensa y el otorgamiento de permisos ha sido satisfecho para el territorio se propone eliminar este indicador.

\section{Indicadores socioculturales}

Relación Visitantes/Población Residente.- Conocer esta relación permite esbozar su tendencia a la frecuentación turística del lugar, así como también a la urbanización y por tanto prever las posibilidades de influencias culturales ajenas a la población residente (Campos-Cámara, 2011). El valor óptimo para este indicador es de $100 \%$ ya que una desviación mayor tendría repercusiones sobre otros índices como el de congestión vehicular, nivel de conflictividad, contaminación acústica y percepción de la comunidad con respecto a la actividad turística. La medición del indicador para el caso del CTSC es del 77.24\%, indicando que la proporción de visitantes con respecto de la población residente aún no es superada y por tanto mantiene otros indicadores dentro del parámetro deseado o permisible. 
Percepción de la comunidad acerca de la actividad turística.- La medición de variables sociales es todo un reto debido a la subjetividad que involucran. Medir las actitudes de una comunidad con respecto a la actividad turística no es una excepción. Una actitud es una "predisposición para responder de una manera consistentemente favorable o desfavorables ante un objeto" (Hernández, 2013:110). El desarrollo sostenible de una comunidad local requiere de la identificación de los impactos sociales del turismo sobre la población residente. Este proceso permite desarrollar estrategias para minimizar el conflicto entre los visitantes y residentes y optimizar oportunidades de desarrollo (Mendoza-Ontiveros et al., 2011). En este contexto, es una realidad que no todos los residentes de una comunidad local reciben beneficios por la actividad turística y por tanto la probabilidad de su participación en el desarrollo del sector puede ser baja (Brougham \& Butler, 1981). La percepción de los residentes de la comunidad local acerca de la actividad arroja luz sobre los intereses reales de la comunidad para su desarrollo, y permite implementar políticas y acciones adecuadas para la planeación turística a nivel local (Mendoza-Ontiveros et al., 2011). Este indicador mostró que el $96.15 \%$ de la población tiene una percepción "positiva" con respecto al CTSC. Sin embargo, enfatizaron que la problemática de la comunidad es la organización social y la carente consciencia del bien común. Si bien esta percepción no afecta la medición del indicador, es importante destacar que los residentes manifiestan que de ser miembros comunitarios colaborativos, responsables y solidarios podrían aprovechar el flujo de visitantes que genera el destino y así mejorar sus ingresos económicos.

Incremento de la conflictividad y el delito en temporada alta.- El incremento de la conflictividad y el delito es un índice que puede concebirse como la sinergia de dos casos. El primero de ellos relacionado al desarrollo turístico como agresiones, hurtos, riñas y vandalismo; y los de mayor envergadura relacionados con la población residente (Ramón-Cardona, 2012). Aunque este índice varia de acuerdo al segmento turístico, lo cierto es que si aumenta considerablemente puede causar un elevado daño en la imagen del lugar y provocar que los visitantes cambien de destino vacacional. El mismo autor menciona también que el tipo de delincuencia que afecta a una población residente y a un destino es la permisividad con los visitantes por dependencia económica y al temor de perder turismo. Aunque es claro que la pequeña delincuencia aumenta con el desarrollo turístico, de acuerdo a la percepción de la población residente no hay una relación estrecha entre la delincuencia y el turismo, sino más bien se encuentra relacionada con el desarrollo económico de la población. Este indicador se encuentra en el valor óptimo para el CTSC. 
Accidentes de tráfico en temporada alta.- De acuerdo a Ramón-Cardona (2012) los residentes de una comunidad tienen mayor posibilidad de adoptar actitudes negativas si el turismo causa congestión o masificación de recursos compartidos, y ello incluye el uso de carreteras. Cuando los accidentes de tráfico tienden aumentar, la población residente es más susceptible a accidentes vehiculares como atropellos y daño en vialidades. Además aumenta la probabilidad de accidentes de tráfico durante la temporada alta. Este indicador obtuvo el valor óptimo, indicando la ausencia de accidentes vehiculares durante la temporada alta.

Nivel de instrucción de los trabajadores de la actividad turística.- Ramón-Cardona (2012) menciona la relación existente entre el nivel educativo de los ciudadanos de la comunidad y los visitantes. En el CTSC las personas con nivel educativo básico mostraron poco interés respecto a la actividad turística, lo que puede estar relacionado con que en la mayoría de los casos se dedican o dedicaban a actividades practicadas tradicionalmente como agricultura, ganadería, extracción de leña y en caso de las mujeres, al trabajo en casa. Aquellos en los que el nivel educativo es medio superior mostraron actitudes proactivas y aprovechan las oportunidades que ofrece el turismo a favor de su desarrollo socioeconómico. Para un nivel educativo superior, las personas se mostraron aun más proactivas y emprendedoras, actitud que se materializa en la ejecución de acciones que permitan mantener al destino en procesos de mejora continua. Un mayor nivel educativo implica no solo mayores salarios sino una mayor participación en el empleo (Instituto Valenciano de Investigaciones Económicas, 2008). Arrow (1997) menciona que la educación tiene un efecto benéfico en las selecciones individuales respecto al mercado laboral y permite adoptar decisiones más eficientes. En el CTSC este indicador se encuentra lejano al valor óptimo, con un valor de $29.5 \%$, indicando que el nivel educativo de la mayoría de los gestores del centro se encuentra en un nivel básico. Un valor bajo en este indicador puede afectar considerablemente los esfuerzos encaminados hacia el alcance de la sostenibilidad por las razones que menciona Ramón-Cardona (2012). La manera en que este indicador puede mejorar su valor es a través de los propios gestores, involucrar a sus hijos y/o familiares con grado de estudios superiores y que les permitan incursionar en conjunto en las actividades y toma de decisiones en el destino.

Indicadores complementarios

Entre los indicadores complementarios, se generaron aquellos que de acuerdo a los propios administradores del centro deben incluirse y medirse periódicamente. En la mayoría de los casos, 
no se cuenta con información suficiente, no obstante, se propone que en cualquier ejercicio similar se incluyan en el entendido de que son estos los indicadores que generan información particular o local para cada destino turístico.

Residuos sólidos generados.- La calidad del ambiente es un elemento indispensable para la gestión del destino, ya que afecta las actitudes de residentes y turistas. Por ello las prácticas deben estar encaminadas hacia la reducción de impactos negativos al ambiente y a la creación de espacios protegidos, tomando en consideración a los residentes (Ramón-Cardona, 2012). Uno de los indicadores clave es la cantidad de residuos sólidos generados, como un indicador resultado del buen manejo de los insumos propios de la actividad turística, así como el efecto positivo de las "buenas prácticas" y "turismo responsable" que ya se solicita a los visitantes al estar en el CTSC. Se propone crear este indicador y estimarlo midiendo la cantidad en kilogramos de basura recolectada en un periodo de tiempo determinado con respecto al periodo anterior. Este indicador en particular, puede ser de fácil aplicación para destinos con características similares al del sitio de estudio.

Condiciones de infraestructura y mobiliario.- Un desarrollo turístico conlleva la creación de infraestructura, oferta comercial y equipamiento, los cuales deben mantenerse en condiciones de calidad para el servicio. Dichos elementos son indispensables en la competitividad y satisfacción de las necesidades y preferencias de los visitantes (Ramón-Cardona, 2012). Este indicador mide las condiciones físicas del mobiliario e infraestructura en el destino. Se obtiene mediante la verificación del inventario total de infraestructura o mobiliario que presenta daños en sus condiciones físicas, con respecto al total disponible en un periodo de tiempo. Se propone realizar brigadas mensuales de monitoreo de sus condiciones en el destino, para mantener información actualizada respecto a este indicador.

Pavimentación de caminos.- La vialidad es un elemento básico para los destinos turísticos, y pueden ser primarias (que atraviesan toda la ciudad), secundarias (relaciona zonas de una ciudad) y terciarias (calles pequeñas). Las vialidades rurales son accesos que unen a comunidades con el mercado regional y son caminos que generalmente carecen de pavimento o tienen una capa delgada de asfalto, son más angostas y con cuestas de mayor inclinación. A la vez, deben presentar buenas 
condiciones y permitir el acceso adecuado a los visitantes y residentes del lugar (Dávila-López, 2015). Para el caso del CTSC los gestores del destino propusieron la pavimentación de la única vía de acceso en respuesta a la demanda de los visitantes. Este indicador mide el porcentaje de camino pavimentado con respecto a su totalidad de la vialidad. El indicador puede ser utilizado en destinos similares al CTSC.

Consumo eléctrico.- La eficiencia energética es el conjunto de acciones que permiten reducir el consumo de energía en los productos y servicios ofertados sin afectar la producción o los niveles de confort de los usuarios o clientes (Instituto Valenciano de Investigaciones Económicas, 2008). Los administradores del CTSC identifican al consumo eléctrico como una problemática de índole económica. Se propone establecer un indicador de consumo eléctrico, que de manera simple mida la cantidad de energía consumida actual en el destino con respecto al periodo anterior. La atención a este indicador implica además el realizar acciones de mejora en el sistema eléctrico y equipamiento, buscando opciones energéticamente más eficientes. De la misma forma, deben realizarse verificaciones mensuales de monitoreo del sistema eléctrico.

Señalización interna.- De acuerdo a la norma mexicana NMX-AA-133-SCFI-2006 sobre requisitos y especificaciones de sustentabilidad del ecoturismo, el mantenimiento de la señalización interna del lugar se debe dar en cumplimento con lo establecido en el Manual de Identidad y Comunicación de la Comisión Nacional de Áreas Naturales Protegidas [CONANP] (Secretaría de Economía, 2006). En el CTSC existe señalética para cada uno de los espacios del sitio, no obstante, no existe un programa de verificación o mantenimiento de la misma. Se propone un indicador de señalización, mismo que mida las condiciones de los letreros en el destino, mediante la verificación del inventario total de señalamientos que se encuentran en mal estado con respecto al total disponible.

\section{Conclusiones}

La selección de indicadores, derivada de la identificación de atributos y temas clave, debe ser un proceso a nivel local. De la misma forma, la inclusión de los administradores o gestores del destino, es fundamental para este proceso. La definición de los indicadores desde una perspectiva local, en acuerdo con propuestas de la propia Organización Mundial de Turismo, es indispensable para identificar problemáticas específicas a cada destino turístico. Incluso, puede permitir depurar 
un sistema de indicadores, manteniendo aquellos más relevantes para un sitio específico. Esta identificación y depuración de indicadores, no puede realizarse de manera correcta si no se considera el papel de los gestores del sitio, pues son ellos quienes poseen información precisa sobre las fortalezas y debilidades propias de un destino específico, además de identificar cuáles indicadores muestran de manera más clara los avances en el mantenimiento de dichas fortalezas y el resarcimiento de las debilidades.

Por otro lado, consideramos que en todo momento deben tomarse en consideración las necesidades y las aspiraciones de la población local hacia la conservación del patrimonio natural y cultural de su lugar de origen, lo que puede generar un compromiso no únicamente con la medición de los indicadores sino con la aplicación de estrategias que eleven la sostenibilidad en la gestión del destino turístico. Una vez identificados los atributos, temas clave, e indicadores, estos pueden ser medidos periódicamente $\mathrm{y}$, en caso de ser necesario, adaptados para ajustarse a nuevos escenarios o realidades.

Para el caso particular del manejo y operación de CTSC, se determinó que no es sostenible en su situación actual. Registramos cinco indicadores con valores en el extremo contrario de los valores óptimos, además de valores diferentes del óptimo en otros indicadores. De manera relevante, el proceso de aplicación de los indicadores, también identificó temas prioritarios que deben ser atendidos para dirigir el manejo y operación del destino a un valor más alto de sostenibilidad de acuerdo a este índice.

Se debe señalar que la ausencia de información para ciertos indicadores, no justifica su ajuste o eliminación para calcular el valor final del índice propuesto; la falta de información es relevante para el resultado final, y en caso de que el manejo del destino incluya la mejora de dichos puntos críticos, un nuevo cálculo del IST debe reflejarlo. La adaptación y uso de esta propuesta puede generar un sistema permanente de diagnóstico que ayude a los administradores del sitio a priorizar temas, de modo que en un caso ideal, no solo sirva para mantener la integridad ambiental del complejo turístico sino también mejore la calidad de vida de las familias que dependen localmente de la actividad turística.

Debe señalarse que el valor ideal de sostenibilidad para esta propuesta, será distinto para otro destino, en función de los indicadores seleccionados y el agregado de indicadores complementarios. No obstante, la estructura general de la propuesta puede replicarse en otros sitios. Es particularmente interesante que la construcción de la propuesta es potencialmente replicable en 
destinos de una misma región o zona, aunque es importante hacer un análisis previo para determinar si la identificación de indicadores será similar, aun tratándose de destinos geográficamente cercanos.

Introducirse al análisis de mercados de sostenibilidad turística requiere de la apertura de paradigmas que van más allá de la aplicación de programas de promoción, satisfacción de los visitantes e inversión en infraestructura (Ibáñez-Pérez, 2012). Requiere de esfuerzos por invertir en la preparación de recursos profesionales en el diagnóstico, planeación, diseño, evaluación y monitoreo de los destinos turísticos para evaluar su avance en el paradigma de la sostenibilidad y competitividad en el tiempo, así como su impacto benéfico en la calidad de vida comunitaria. También debe constituir modificaciones en los paradigmas de planeación turística y abordar en medida de lo posible la intervención holística de actores sociales.

Evaluar un desarrollo turístico a través de un sistema de indicadores de sostenibilidad permite el diálogo entre gestores de la actividad, la comunidad local, diseñadores de políticas ante las necesidades, prioridades y demandas ambientales, económicas, sociales y culturales en el destino (Ibáñez-Pérez, 2012). Si se toman en cuenta estos puntos es posible realizar comparaciones y trabajar en el establecimiento de políticas públicas, estándares de calidad, procesos de desarrollo y planeación de los destinos ecoturísticos in situ y ex situ.

La evaluación de la sostenibilidad del CTSC a través de indicadores apunta que se requiere de esfuerzos compartidos en los procesos de planeación, en las capacidades organizativas de los gestores, así como en la generación de bancos de información básica a nivel local, mismos que permitan conocer las tendencias en el tiempo de la actividad turística y de la inclusión de la comunidad local.

\section{Referencias bibliográficas}

Alarcón Hernández, P. (2010). Implicaciones y contradicciones del ecoturismo en La sima de las cotorras, Ocozocoautla de Espinoza, Chiapas. El Colegio de la Frontera Sur, San Cristóbal de las Casas, Chiapas.

Arrow, K. J. (1997). The benefits of education and the formation of preferences. In The Social Benefits of Education (1st ed., pp. 11-16). Michigan: University of Michigan Press.

Ávila-Bercial, Á., \& Barrado-Timón, D. A. (2005). Nuevas tendencias en el desarrollo de destinos turísticos: marcos conceptuales y operativos para su planificación y gestión. Cuadernos de Turismo, $0(15), 27-44$.

Ayuso Siart, S. (2003, September 15). Gestión sostenible en la industria turística. Retórica y práctica en el sector hotelero español ( $\mathrm{PhD}$ thesis). Universitat Autónoma de Barcelona. Disponible en http://www.tdx.cat/handle/10803/4954?show=full 
Barber, J. R., Crooks, K. R., \& Fristrup, K. M. (2010). The costs of chronic noise exposure for terrestrial organisms. Trends in Ecology \& Evolution, 25(3), 180-189. DOIR: https://doi.org/10.1016/j.tree.2009.08.002

Benur, A. M., \& Bramwell, B. (2015). Tourism product development and product diversification in destinations. Tourism Management, 50, 213-224. DOI: https://doi.org/10.1016/j.tourman.2015.02.005

Blancas-Peral, F. J., González-Lozano, M., Guerrero-Casas, F. M., \& Lozano-Oyola, M. (2010). Indicadores sintéticos de turismo sostenible: una aplicación para los destinos turísticos de Andalucía. Revista Electrónica de Comunicaciones Y Trabajos de ASEPUMA, 11(1), 85. DOI: https://doi.org/https://dialnet.unirioja.es/descarga/articulo/3674366.pdf

Blanco-Cerradelo, L., Diéguez-Castrillón, M. I., \& Gueimonde-Canto, A. (2015). Propuesta de indicadores de recursos de competitividad turística en los espacios naturales protegidos. PASOS. Revista de Turismo Y Patrimonio Cultural, 13(4), 947-957.

Bolwell, D., \& Weinz, W. (2008). Guide for social dialogue in the tourism industry (ILO Working Paper No. 994246583402676). International Labour Organization. Disponible en https://ideas.repec.org/p/ilo/ilowps/994246583402676.html

Brougham, J. E., \& Butler, R. W. (1981). A segmentation analysis of resident attitudes to the social impact of tourism. Annals of Tourism Research, 8(4), 569-590. DOI: https://doi.org/10.1016/01607383(81)90042-6

Camacho-Ruiz, E., Carrillo-Reyes, A., Rioja-Paradela, T. M., \& Espinoza-Medinilla, E. E. (2016). Indicadores de sostenibilidad para el ecoturismo en México: estado actual. LiminaR. Estudios Sociales y Humanísticos, 14(1), 156-168.

Campos-Cámara, B. L. (2011). Presión turística y urbanística: Vulnerables al cambio climático en el Caribe Mexicano. Quivera, 13(2), 1-13.

Cano-Contreras, E. J., Medinaceli, A., Diago, O. L. S., \& Villamar, A. A. (2015). Código de Ética para la Investigación, La Investigación-Acción y la Colaboración Etnocientífica en América Latina. Versión Uno. Etnobiología, 12(4), 5-6.

Carruitero, P. B. (2011). Estacionalidad de la demanda de turismo en Argentina (MSc thesis). Facultad de Ciencias Económicas, La Plata. Disponible en http://hdl.handle.net/10915/3472

Chuvieco, E. (1998). El factor temporal en teledetección: evolución fenomenológica y análisis de cambios | Asociación Española de Teledetección. Revista AET, 10, 1-9.

Conferencia de las Naciones Unidas sobre Comercio y Desarrollo. (2013). Turismo sostenible: contribución del turismo al crecimiento económico y al desarrollo sostenible (Contribución del turismo al desarrollo sostenible No. TD/B/C.I/EM.5/2) (p. 22). Naciones Unidas. Disponible en http://unctad.org/meetings/es/SessionalDocuments/ciem5d2_sp.pdf

Dávila-López, A. (2015). Centros Integralmente Planeados (CIPS) en México: el proyecto turístico del FONATUR. QRU: Quaderns de Recerca en Urbanisme, (5/6), 270-285.

Galle, R. E. (2004). El nivel de ocupación en turismo. Facultad de Ciencias Económicas U.N.L.P. Disponible en http://eco.unne.edu.ar/contabilidad/costos/iapuco/trabajo05_iapuco.pdf

Gleason, J. A. (2005). Manual de aprovechamiento de aguas pluviales en centros urbanos (1st ed.). Guadalajara: Universidad de Guadalajara. Disponible en https://www.iberlibro.com/Manualaprovechamiento-aguas-pluviales-centros-urbanos/5779911495/bd

Gössling, S., Peeters, P., Hall, C. M., Ceron, J.-P., Dubois, G., Lehmann, L. V., \& Scott, D. (2012). Tourism and water use: Supply, demand, and security. An international review. Tourism Management, 33(1), 1-15. DOI: https://doi.org/10.1016/j.tourman.2011.03.015

Habibullah, M. S., Din, B. H., Chong, C. W., \& Radam, A. (2016). Tourism and Biodiversity Loss: Implications for Business Sustainability. Procedia Economics and Finance, 35, 166-172. DOI: 
InterSedes, N44. Vol XXI (2020). ISSN 2215-2458

https://doi.org/10.1016/S2212-5671(16)00021-6

Hassan, S. S. (2000). Determinants of Market Competitiveness in an Environmentally Sustainable Tourism Industry. Journal of Travel Research, 38(3), 239-245. DOI: https://doi.org/10.1177/004728750003800305

Hernández, R. (2013). Metodologia de la Investigacion (6th ed.). México: McGraw Hill.

Herrero, L. M. J. (2017). Desarrollo sostenible: Transición hacia la coevolución global (1st ed.). Madrid: Ediciones Pirámide.

Ibáñez-Pérez, R. M. (2012). Indicadores de sustentabilidad: utilidad y limitaciones. Teoría y Praxis, (11), $102-126$.

Iglesias, C., Mata, C., \& Malo, J. E. (2012). The Influence of Traffic Noise on Vertebrate Road Crossing Through Underpasses. Ambio, 41(2), 193-201. DOI: https://doi.org/10.1007/s13280-011-0145-5

Instituto Nacional de Estadística y Geografía. (2010). Censo de Población y Vivienda 2010. Disponible en http://www.censo2010.org.mx/

Instituto Valenciano de Investigaciones Económicas. (2008). Fundación Bancaja: Publicaciones : Efectos del nivel educativo sobre las probabilidades de empleo. Bancaja. Disponible en http://www.fundacionbancaja.es/jovenes/publicaciones/cuadernos-de-capital-humano-yempleo/89-efectos-del-nivel-educativo-sobre-las-probabilidades-de-empleo.aspx

Kniffki, J., Calero, A., \& Castillo, R. (2009). Metodología comunitaria para el desarrollo social (1st ed.). Managua: Editorial Don Bosco.

Lemus, J., \& Pérez, E. (2011). La percepción de las condiciones sociales y su influencia en el desarrollo de la actividad turística en la parroquia Higuerote, Estado Miranda, Venezuela. Terra. Nueva Etapa, 27(41), 97-123.

Luffiego-García, M., \& Rabadán-Vergara, J. M. (2000). La evolución del concepto de sostenibilidad y su introducción en la enseñanza. Enseñanza de las ciencias: revista de investigación y experiencias didácticas, 18(3), 473-486.

Méndez-Velandia, C. A. (2013). La contaminación visual de espacios públicos en Venezuela. Revista Gestión Y Ambiente, 16(1), 45-60.

Mendoza-Ontiveros, M. M., Monterrubio-Cordero, J. C., \& Fernández-Aldecua, M. J. (2011). Impactos sociales del turismo en el Centro Integralmente Planeado. Gestión Turística, 15, 47-73.

Mihalič, T. (2000). Environmental management of a tourist destination: A factor of tourism competitiveness. Tourism Management, 21(1), 65-78. DOI: https://doi.org/10.1016/S02615177(99)00096-5

Neibaur, E. E., \& Anderson, E. P. (2016). An examination of factors affecting sustainability of domestic rainwater harvesting systems in a rural, semi-arid region of Mexico. Water Science and Technology: Water Supply, ws2016066. DOI: https://doi.org/10.2166/ws.2016.066

Pavón, R. S., Muñoz, A. P., \& Arcos, L. A. (2007). Medición de la capacidad de carga turística de Cozumel. El Periplo Sustentable, (13), 33-61.

Pérez Albert, Y., \& Nel-Io Endreu, M. (2013). Propuesta de indicadores para evaluar la sostenibilidad de la actividad turística. El caso del Valle de Viñales (Cuba). Anales de geografía de la Universidad Complutense, 33(1), 193-210.

Punzo, L. F. (2004). Sostenibilidad del turismo y desarrollo económico local: El caso de la región de Toscana, España. Cuaderno Virtual de Turismo, 4, 1-30.

Ramón-Cardona, J. (2012, December 19). Actitudes de los residentes hacia el turismo en destinos turísticos consolidados: El caso de Ibiza (PhD thesis). Universitat de les Illes Balears. Disponible en http://www.tdx.cat/handle/10803/104266

Rivas García, J., \& Magadán Díaz, M. (2007). Los Indicadores de Sostenibilidad en el Turismo. Revista de Economía, Sociedad, Turismo Y Medio Ambiente, (6), 27-61. 
Rivas, H. (1998). Los impactos ambientales en áreas turísticas rurales y propuestas para la sustentabilidad. Gestión Turística, (3), 47-75.

Secretaría de Economía. Norma Oficial Mexicana NMX-AA-133-SCFI-2006 que establece los requisitos y especificaciones de sustentabilidad del ecoturismo, Pub. L. No. NMX-AA-133-SCFI-2006 (2006).

Secretaría de Medio Ambiente y Recursos Naturales. (2001). Programa de manejo Reserva de la Biósfera Selva el Ocote. Comisión Nacional de Áreas Naturales Protegidas. Disponible en http://www.conanp.gob.mx/que_hacemos/pdf/programas_manejo/ocote.pdf

Solari, F. A., \& Cazorla, L. (2009). Valoración de la calidad y fragilidad visual del paisaje. Cuadernos Del Centro de Estudios de Diseño Y Comunicación, 10(30), 213-226.

Stansfeld, S. A., \& Matheson, M. P. (2003). Noise pollution: non-auditory effects on health. British Medical Bulletin, 68(1), 243-257. DOI: https://doi.org/10.1093/bmb/ldg033

Vanegas-Montes, G. M. (2006). Ecoturismo, instrumento de desarrollo sostenible (Undergraduate thesis). Universidad de Antioquia, Medellín.

Vera-Rebollo, J. F., \& Baidal, J. A. I. (2003). Sistema de indicadores aplicado a la planificación y gestión del desarrollo turístico sostenible. In L. Valdés Peláez, J. M. Pérez Fernández, \& E. A. del Valle Tuero (Eds.), Experiencias públicas y privadas en el desarrollo de un modelo de turismo sostenible (1st ed., pp. 105-129). Oviedo, España: Oviedo: Fundación Universidad de Oviedo. Disponible en https://dialnet.unirioja.es/servlet/articulo?codigo $=3148624$

World Tourism Organization. (1996). What Tourism Managers Need to Know. Madrid: World Tourism Organization. DOI: https://doi.org/10.18111/9789284401505

World Tourism Organization. (2004). Indicators of Sustainable Development for Tourism Destinations A Guidebook (1st ed.). Madrid, España: World Tourism Organization. Disponible en http://www.eunwto.org/doi/book/10.18111/9789284407262 